\title{
Calidad de vida general, depresión y ansiedad: diferencias de acuerdo con el control del asma
}

\author{
General Quality of Life, Depression, and Anxiety: \\ Differences According to Asthma Control
}

\author{
Cintia Tamara Sánchez Cervantes ${ }^{1}$ \\ https://orcid.org/0000-0001-9365-6233 \\ Centli Guillén-Díaz-Barriga² \\ https://orcid.org/0000-0001-6972-4259 \\ Violeta Alejandra Mendoza Madrigal ${ }^{3}$ \\ D https://orcid.org/0000-0003-4347-6642
}

${ }^{1}$ Servicio de Salud Mental, Hospital Juárez, México

2 Área de Investigaciones Epidemiológicas y Psicosociales, Instituto Nacional de Psiquiatría Ramón de la Fuente Muñiz, México ${ }^{3}$ Facultad de Estudios Superiores Iztacala, Universidad Nacional Autónoma de México, México

Resumen. Objetivo. Comparar la calidad de vida (CdV) evaluada a través del Inventario de Calidad de Vida y Salud, así como depresión y ansiedad entre pacientes con asma controlada y asma no controlada. Método. Es un estudio transversal, ex post facto en el que participaron 149 adultos mexicanos con asma. Resultados. Se muestran diferencias significativas con tamaños del efecto de moderados a grandes en las sub-escalas de desempeño físico, aislamiento, tiempo libre, vida cotidiana, ansiedad y depresión. Las áreas que correlacionaron significativa y positivamente fueron vida cotidiana con aislamiento y tiempo libre, depresión con aislamiento y vida cotidiana. Pacientes con asma no controlada presentaron afectación en las sub-escalas de CdV relacionadas con actividades físicas y sociales, intervenciones psicológicas deben atender a estas áreas.

Palabras clave. Asma bronquial, adultos, InCaViSa, actividades físicas, actividades sociales.

Abstract. Objective. Compare quality of life (QoL) evaluated through the Quality of Life and Health Inventory, as well as depression and anxiety between subjects with controlled asthma and uncontrolled asthma. Method. This is a Cross-sectional, ex post facto study involving 149 Mexican adults with asthma. Results. Significant differences with moderate to large effect size were found in these sub-scales such as physical performance, isolation, free time, daily life, anxiety, and depression. The areas that correlated significantly and positively were daily life with isolation and free time, depression with isolation, and daily life. Subjects with uncontrolled asthma are affected in the QoL subscales related to physical and social activities, psychological treatments must attend to these areas.

Keywords. Bronchial Asthma, Adults, InCaViSa, Physical Activities, Social Activities.

${ }^{1}$ Cintia Tamara Sánchez Cervantes. Servicio de Salud Mental, Hospital Juárez de México, México. Dirección postal: Avenida Instituto Politécnico Nacional \#5160, Colonia Magdalena de las Salinas, Gustavo A. Madero, C.P. 07760. Ciudad de México. E-mail: cintiacervantess@gmail.com

${ }^{2}$ Centli Guillén-Díaz-Barriga. Área de Investigaciones Epidemiológicas y Psicosociales, Instituto Nacional de Psiquiatría Ramón de la Fuente Muñiz, México. E-mail: centligdb@gmail.com

${ }^{3}$ Violeta Alejandra Mendoza Madrigal. Facultad de Estudios Superiores Iztacala, Universidad Nacional Autónoma de México, México. E-mail: violeta_alejandra_04@hotmail.com

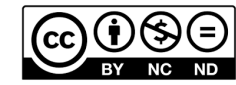

Esta obra está bajo una licencia de Creative Commons Reconocimiento-NoComercial-SinObraDerivada 4.0 Internacional. 


\section{Introducción}

La Iniciativa Global del Asma (2021; GINA, por sus siglas en inglés) define el asma como una enfermedad de origen heterogéneo, cuya principal característica es la inflamación crónica de la vía aérea. Se caracteriza por una historia de síntomas respiratorios que varían en el tiempo e intensidad tales como silbidos, falta de aire, opresión torácica y tos, junto con la limitación del flujo de aire espiratorio.

El tratamiento para el asma busca el control de los síntomas y la reducción de exacerbaciones asmáticas para que así el paciente tenga la mejor calidad de vida posible (GINA, 2021). Los individuos con asma controlada (es decir, mejor funcionamiento físico general y nula o mínima necesidad del uso de tratamientos de rescate) tienen niveles de calidad de vida similares a los pacientes sin enfermedades crónicas (Jansson et al., 2016). Sin embargo, investigaciones muestran que en la realidad los pacientes con asma, en un $50 \%$ tienen un mal control de la enfermedad y el $75 \%$ tiene mala adherencia al tratamiento (GINA, 2021) además de reportar niveles de calidad de vida deficientes (Lomper et al., 2016).

Relacionado a lo anterior, se ha documentado ampliamente que el control de la enfermedad y la calidad de vida se encuentran correlacionadas significativamente (González-Barcala et al., 2012; González-Feire et al., 2019; Hossny et al., 2017). Se sostiene que los pacientes con asma clasificada como no controlada son aquellos que presentan niveles más bajos de calidad de vida (Ilmarinen et al., 2019).

Al considerar la severidad, el grado de control de la enfermedad es el parámetro clínico más importante para determinar la calidad de vida general $(\mathrm{CdV})$ o la calidad de vida relacionada con el asma (CV-RA; Uchmanowicz et al., 2016). Ya sea que el control de la enfermedad sea definido por variables objetivas, como resultados de espirometría o valores de flujometría o por aspectos subjetivos como el auto-reporte en cuestionarios de control de la enfermedad.

Es importante señalar que la CdV es un constructo multifactorial complejo, cuyo objetivo y subjetivo se conjugan. Principalmente, abarca las áreas física, psicológica y social, por lo que es un concepto que se modifica de acuerdo con el estado emocional, las percepciones y las expectativas del individuo. No es un concepto estático, sino como algo dependiente del tiempo, que sufre cambios y refleja las experiencias de la vida (Urzúa \& Caqueo-Urízar, 2012). La CdV se compone de diversas variables objetivas y subjetivas; factores como calidad medio-ambiental, ingreso econónomico, y la propiedad de bienes y servicios públicos, son considerados como variables objetivas (Sánchez-Sosa \& GonzálezCelis, 2006). Mientras que Fernández-Ballesteros (1997) considera que las subjetivas (o personales) abarcan todas aquellas que se relacionan con la percepción: proceso de saludenfermedad, las relaciones interpersonales, participación en actividades de ocio, bienestar y satisfacción con la vida.

En consecuencia, es importante evaluar la calidad de vida del paciente además de los síntomas propios de la enfermedad, para obtener información completa sobre el estado de salud de los pacientes (Coban \& Aydemir, 2014; Hossny et al., 2017; Ilmarien et al., 2019).

Además del control de la enfermedad, se ha determinado que el estado de ánimo negativo es una de las variables que afecta significativamente la CdV (Coban \& Ediger, 2018; Ekici et al., 2006). Se ha reportado que, si bien, los estados de ansiedad, depresión y un enfrentamiento a los problemas de estilo centrado en la emoción no son la causa, se 
han encontrado correlacionados con el asma no controlada y una CdV con niveles más bajos (González-Freire et al., 2019). Por ejemplo, con datos derivados de un instrumento enfocado a la CV-RA Méndez-Guerra et al. (2003) concluyen que en la población mexicana con asma, el área que evalúa la función emocional es la que se suele ver con mayor afectación, principalmente en pacientes con asma clasificada como no controlada o severa.

Aunque una medida de CV-RA nos puede dar información respecto a la enfermedad, autores como Guillén et al. (2020) argumentan que el uso de estas medidas no se encuentra adaptadas a la cultura mexicana y pueden resultar insuficientes para evaluar los beneficios que se derivan de intervenciones psicológicas. Las autoras indican que al tratarse de instrumentos tan específicos, se centran más en la sintomatología física y no se evalúan los aspectos subjetivos del individuo.

Instrumentos como el Inventario de Calidad de Vida y Salud (InCaViSa), pueden ampliar el panorama y resultar extremadamente útiles tanto en la investigación clínica como experimental, ya que se hace una evaluación general de la C $\mathrm{V} V$ y fue construido con población de pacientes mexicanos con enfermedades crónicas. Además, estos instrumentos tienen la ventaja de haber sido diseñados con la intención de evaluar diversas áreas de la vida de pacientes que padecen enfermedades crónicas (Riveros et al., 2009). Este instrumento ha aportado datos referentes al tratamiento con pacientes que padecen hipertensión arterial (Riveros, Ceballos et al., 2005) y diabetes mellitus (Riveros, Cortazar-Palapa et al., 2005).

En el caso del asma, el InCaViSa fue empleado por Juárez (2010) para evaluar el impacto de una intervención cognitivo-conductual dirigida a la adherencia terapéutica de nueve mujeres mexicanas con diagnóstico de asma; sin embargo, hasta el momento no se tiene reporte de que haya sido empleado para describir las características generales de una muestra amplia.

Contar con datos proporcionados por el InCaViSa puede ser beneficioso para conocer a profundidad las áreas en que se están viendo afectados los pacientes, lo cual depende del control de su enfermedad y permite diseñar intervenciones dirigidas hacia estas áreas específicas. De igual forma, tener conocimiento de las áreas que están siendo afectadas por los pacientes que presentan ansiedad y/o depresión, servirán para proponer planes de acción. Los datos aquí generados podrían complementar la investigación de Méndez-Guerra et al. (2003).

Se espera que el grado de control del asma produzca efectos sobre la CdV, la ansiedad y la depresión (Ilmarinen et al., 2019). Por lo tanto, el objetivo general del presente trabajo es comparar la calidad de vida (evaluada con el InCaViSa) entre los pacientes con asma controlada y asma no controlada. Como objetivo particular, la investigación buscó conocer la asociación entre los niveles de ansiedad, depresión y las áreas calidad de vida, así como el control de la enfermedad.

\section{Método}

\section{Participantes}

Se trató de un muestreo no probabilístico por conveniencia. El estudio se llevó a cabo en la sala de espera de la clínica de asma del INER, la cual es un área común, iluminada y ventilada, con una capacidad aproximada de 30 pacientes. Los 159 participantes fueron pacientes con asma usuarios de la Clínica de Asma del Instituto Nacional de Enfermedades Respiratorias "Ismael Cosío Villegas", INER. Previo a su implementación, el protocolo fue aprobado por el comité ético y científico del instituto (DI/CCB/292/11; Código C46-11). 
La selección de participantes se basó en criterios de inclusión: (a) diagnóstico de asma confirmado por neumólogo del instituto, (b) edad mínima de 18 años, (c) saber leer y escribir y (d) firmar el consentimiento informado; criterios de exclusión: (a) enfermedad psiquiátrica o condición cognitiva que impidieron contestar la batería de pruebas y (b) no desear participar en el estudio. Y criterios de eliminación: (a) dejar inconclusos los instrumentos de evaluación (25\%) o (b) contestarlos de manera no correspondiente a las instrucciones.

\section{Procedimiento}

Todos los pacientes participaron de forma informada, consentida y voluntaria. Los pacientes fueron reclutados en la sala de espera y se identificaban mediante la lista diaria de consulta externa proporcionada por el servicio de enfermería. Mientras los pacientes esperaban ser llamados a su cita con el médico eran abordados por alguno de los responsables de la investigación. De manera individual se les explicó los objetivos de la investigación y se extendió la invitación a participar en el proyecto. Se brindó el consentimiento informado para su lectura, y en caso de acceder, el participante procedía a firmar. El investigador brindó los instrumentos, explicó su llenado y dejó que el participante los llenara de forma autónoma. En caso de existir dudas, el participante podía consultar al investigador, el cual aguardaba en el área de la sala de espera.

\section{Instrumentos}

Cuestionario de datos sociodemográficos y autoevaluación de apego. Documento con el que se recopilaron las variables de edad, sexo, estado civil, escolaridad, ocupación y se solicitaba al paciente hacer una autoevaluación de su apego al tratamiento, con un puntaje del cero a diez, en el que a mayor puntaje significa un mejor apego al tratamiento.

Cuestionario de Control de Asma (Asthma Control Test, ACT por sus siglas en inglés). Cuestionario diseñado por Nathan et al. (2004) basado en criterios internacionales, el cual se compone de cinco preguntas que evalúan la percepción del paciente acerca del grado de control de su enfermedad cuyo puntaje deriva a tres categorías: puntajes de 20-25 son clasificados como asma controlada, de 16-19 asma parcialmente controlada y de 5 a 15 puntos asma mal controlada. Se ha reportado una confiabilidad de $\alpha=.77$ en población mexicana (Guillén \& González-Celis, 2019). La Guía Mexicana del Asma (LarenasLinnemann et al., 2017) recomienda su uso para evaluar el control de la enfermedad, ya que se trata de una herramienta breve y sencilla que brinda información certera en referencia al control de la enfermedad. En la presente investigación se decidió recategorizar de uno a 19 puntos como asma no controlada y considerar de 20 a 25 puntos como asma controlada. Esta categorización se deriva de la necesidad de operazionalizar la conducta para visualizarla en términos de su ocurrencia/ausencia. Entonces, se busca la presencia o ausencia y no la parcialidad del control de la enfermedad.

Inventario de Depresión de Beck (IDB). Herramienta de evaluación autoadministrable de 21 reactivos que evalúan la gravedad de la sintomatología depresiva. Cada reactivo se califica del cero al tres, indicando a mayor puntaje mayor severidad del síntoma de depresión. A partir de los 17 puntos se considera como una calificación indicativa de depresión moderada y clínicamente relevante. Se encuentra adaptado y estandarizado para población mexicana con un índice $\alpha=.87$ (Jurado et al., 1998).

Inventario de ansiedad de Beck (IAB). Conformado por 21 reactivos que piden a los respondientes indicar cuánto han sido afectados por una serie de síntomas durante la última semana; en una escala de cero a tres, en el que cero significa "poco o nada" y tres "severamente". El puntaje total se obtiene de la suma de cada uno de los reactivos, siendo el 
puntaje mínimo cero y el máximo 63. El punto de corte para considerar ansiedad clínicamente relevante es de 16 puntos. Su índice de confiabilidad es $\alpha=.84$ (Robles et al., 2001).

Inventario de Calidad de Vida y Salud (InCaViSa). Elaborado por Riveros et al. (2009), cuenta con 53 reactivos en escala tipo Likert con seis opciones de respuesta que van de cero a cinco agrupados en las siguientes áreas o sub-escalas: desempeño físico, familia, redes sociales, relación con el médico, preocupaciones, aislamiento, dependencia médica, percepción corporal, funciones cognitivas, actitud ante el tratamiento, tiempo libre y vida cotidiana, la máxima calificación que se puede alcanzar por área es de 20 y la mínima de cero. Para la presente investigación, se ordenó el sentido de todas las sub-escalas hacia la misma dirección, por lo que, a mayor puntaje, mayor calidad de vida. Además, contiene una escala visual análoga (EVA) en la que el paciente otorga una calificación a tres parámetros: (a) calidad de vida en general; (b) salud en general y (c) su salud en comparación al último año. La EVA se puede calificar en una escala del cero al cinco, en la que a mayor puntuación se refleja una mejor calidad de vida.

En la parte final del instrumento se enlista una serie de síntomas físicos (dolor de cabeza, dolor muscular, dolor articular, hinchazón de tobillos y/o piernas, problemas de la vista, problemas para oír, comezón o lesiones en la piel, tos, problemas urinarios, tics, y mal olor) a los que se le otorga una calificación de frecuencia que va del cero al cinco, en el que un puntaje más alto significa mayor frecuencia de presentar el síntoma.

Los valores alpha de Cronbach oscilan entre .93 para el área de vida cotidiana y .68 para actitud ante el tratamiento. El análisis factorial arrojó que cada área se compuso por cuatro reactivos y explicaron el 68\% de la varianza.

El InCaViSa no otorga una calificación total, sino una calificación por cada sub-escala, ya que los autores alientan a precisar las áreas que merecen especial atención y fijar metas específicas de tratamiento.

Análisis de datos

Los datos obtenidos fueron codificados y procesados en el paquete estadístico SPSS, versión 20. Se empleó estadística descriptiva (frecuencias y porcentajes) para las variables sociodemográficas.

Para los análisis del InCaViSa, las sub-escalas que denotaban características negativas en su título (preocupaciones, aislamiento y dependencia médica) o que sus reactivos estuvieran redactados con connotación negativa (percepción corporal, funciones cognitivas, actitud ante el tratamiento, tiempo libre y vida cotidiana) fueron invertidos en sus valores, esto con el fin de que todas las áreas conservaran la misma dirección. Es decir, que a mayor puntaje se refleje una mejor calidad de vida.

Los datos no se distribuyeron de forma normal, por lo tanto, se empleó la prueba $t$ de Student acompañada de la corrección por medio de bootstrapping. Con esta prueba, se realizaron las comparaciones entre los grupos de pacientes con asma no controlada (calificación en ACT $\leq 19$ puntos) y los pacientes con asma controlada (puntaje del ACT $\geq 20$ ), en relación con las áreas que evalúa el InCaViSa y los puntajes de IAB, IDB. En aquellas variables en las que se detectaron diferencias estadísticamente significativas, se calculó la $d$ de Cohen para medir el tamaño del efecto. Finalmente, para las asociaciones entre los valores obtenidos en las pruebas IDB, IAB, ACT y las áreas del InCaViSa se empleó el coeficiente $r$ de Pearson. 


\section{Resultados}

Se reclutaron un total de 159 pacientes, de los cuales 10 dejaron incompleta más del $25 \%$ de la batería, por lo tanto, se eliminaron del análisis. Se presentan los datos de los 149 usuarios que completaron las evaluaciones.

La muestra fue conformada en su mayoría por mujeres (71.1\%) y la media de edad fue de 41.45 años ( $D T=13.62)$. La mayoría contaba con un nivel académico de secundaria (25\%) y se dedicaban a labores del hogar (35.4\%). El 70\% clasificó su asma como no controlada. Estimaron su apego al tratamiento del asma con una calificación promedio de 7.83. Del total de los participantes, el $68.5 \%$ presentaban algunas co-morbilidades derivadas del asma o diferente a esta (Tabla 1).

Se presenta la comparación entre pacientes con asma controlada y no controlada en las áreas del InCaViSa, depresión y ansiedad (Tabla 2). Se muestra que el control del asma establece diferencias estadísticamente entre grupos en las sub-escalas del InCaViSa de: desempeño físico $(t(147)=4.216 ; p<.002 ; d=.742)$, aislamiento $(t(147)=2.882 ; p=$ $.005 ; d=.523)$, percepción corporal $(t(147)=3.697, p=.003 ; d=.051)$, actitud ante el tratamiento $(t(147)=2.611 ; p=.008 ; d=.409)$, tiempo libre $(t(147)=2.917 ; p=.008 ; d$ $=.514)$, y vida cotidiana ( $t(147)=3.929 ; p=.002 ; d=.514)$. Así como diferencias entre grupos en la EVA de calidad de vida $(t(136)=2.883 ; p=.007 ; d=.406)$ y la EVA de la calidad de vida en relación con el año anterior $(t(144)=2.564 ; p<.016$; $d=.525)$. En referencia al tamaño del efecto, los valores se ubicaron por arriba de moderado ( $d$ $<0.5)$, a excepción de percepción corporal y EVA de calidad de vida. Se encontraron diferencias significativas en el nivel de ansiedad $(t(144.1)=6.481 ; p=.001 ; d=1.026)$ y depresión $(t(128.9)=4.082 ; p=.001 ; d=.639)$, ambas con un tamaño del efecto de moderado a grande.

Se muestra la comparación de síntomas físicos enlistados en el InCaViSa entre pacientes con control de la enfermedad y no control (Tabla 3). Se presentan las diferencias entre grupos, existieron diferencias estadísticamente significativas con tamaños del efecto moderados para dolor de cabeza $(t(112.6)=3.257 ; p=.002 ; d=.562)$, dolor muscular $(t(145)=3.832 ; p=.004 ; d=.559)$, dolor articular $(t(107.7)=3.832 ; p=.004 ; d=.641)$. Mientras que resultaron significativos, pero con tamaños del efecto bajo: problemas urinarios $(t(140.1)=2.191 ; p=.033 ; d=.336)$. Se reportó un tamaño del efecto grande en el síntoma de tos $(t(141.8)=7.248 ; p=.001 ; d=1.06)$.

En la Tabla 4 se muestran las correlaciones entre las doce áreas de InCaViSa, IAB, IDB y $A C T$. Las áreas que correlacionaron significativamente y fuerte fueron percepción corporal y aislamiento $(r=.69 ; p<.01)$, tiempo libre y aislamiento $(r=.59 ; p<.01)$, vida cotidiana y aislamiento $(r=.78 ; p<.01)$, percepción corporal con vida cotidiana $(r=$ $.64 ; p<.01)$, tiempo libre y actitud ante el tratamiento $(r=-.61, p<.01)$, vida cotidiana y actitud ante el tratamiento $(r=.60 ; p<.01)$, y finalmente, vida cotidiana y tiempo libre $(r=.77 ; p<.01)$.

En la Tabla 5 se muestra que al identificar la asociación de los puntajes obtenidos con la medida InCaViSa total y en sus áreas, se encontró que el área que tuvo una correlación significativa alta fue la de vida cotidiana $(r=.62 ; p<.01)$. Por otra parte, el IDB correlacionó de forma alta y negativa con el área de aislamiento $(r=-.64 ; p<.01)$ y con vida cotidiana $(r=-.65 ; p<.01)$. Las áreas del InCaViSa restantes presentaron correlaciones inexistentes, por lo que no se representan en la tabla. 
Tabla 1

Características generales de los participantes

\begin{tabular}{|c|c|c|}
\hline Variables & $n$ & $\%$ \\
\hline \multicolumn{3}{|l|}{ Sexo } \\
\hline Mujeres & 106 & 71.1 \\
\hline Hombres & 43 & 28.9 \\
\hline \multicolumn{3}{|l|}{ Estado Civil } \\
\hline Soltero & 50 & 34.7 \\
\hline Casado & 76 & 52.8 \\
\hline Divorciado & 9 & 6.3 \\
\hline Viudo & 5 & 1.9 \\
\hline No especifica & 7 & 4.3 \\
\hline \multicolumn{3}{|l|}{ Escolaridad } \\
\hline Sin educación formal & 4 & 2.7 \\
\hline Primaria & 32 & 21.5 \\
\hline Secundaria & 33 & 22.1 \\
\hline Preparatoria & 31 & 20.8 \\
\hline Licenciatura & 29 & 19.5 \\
\hline Posgrado & 6 & 4 \\
\hline No especifica & 14 & 9.4 \\
\hline \multicolumn{3}{|l|}{ Ocupación } \\
\hline Ama de casa & 50 & 33.6 \\
\hline Profesionista & 20 & 13.9 \\
\hline Empleada doméstica & 17 & 11.4 \\
\hline Estudiante & 16 & 10.7 \\
\hline Comerciante & 14 & 9.4 \\
\hline Oficios & 7 & 4.7 \\
\hline Otro & 25 & 16.79 \\
\hline \multicolumn{3}{|l|}{ Control de la enfermedad } \\
\hline Asma no controlada & 104 & 69.79 \\
\hline Asma controlada & 45 & 30.21 \\
\hline \multicolumn{3}{|c|}{ Presencia de comorbilidades } \\
\hline Sí & 102 & 68.5 \\
\hline No & 47 & 31.5 \\
\hline
\end{tabular}

Actualidades en Psicología, 35(131), 2021, 35-51 


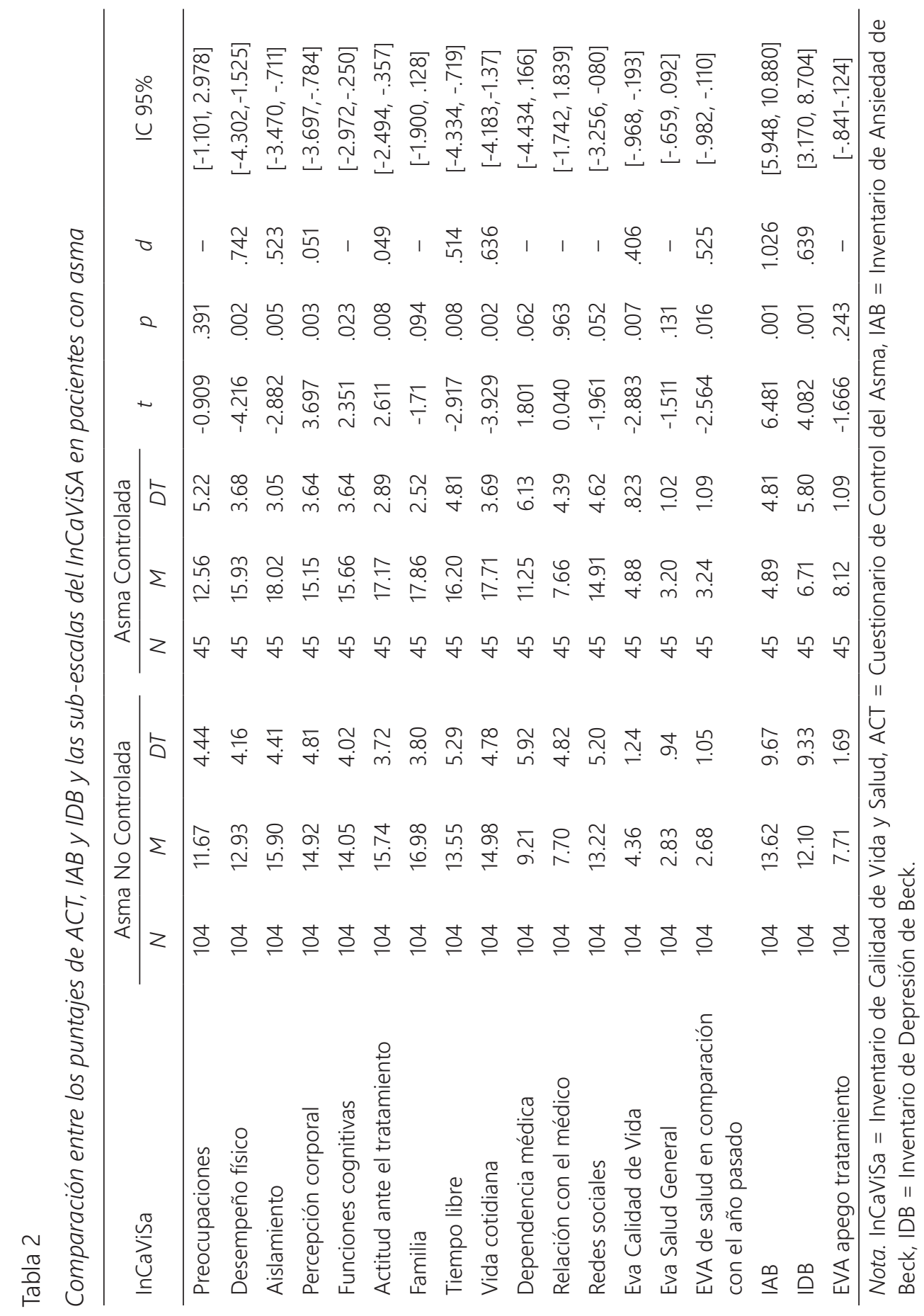

Actualidades en Psicología, 35(131), 2021, 35-51 


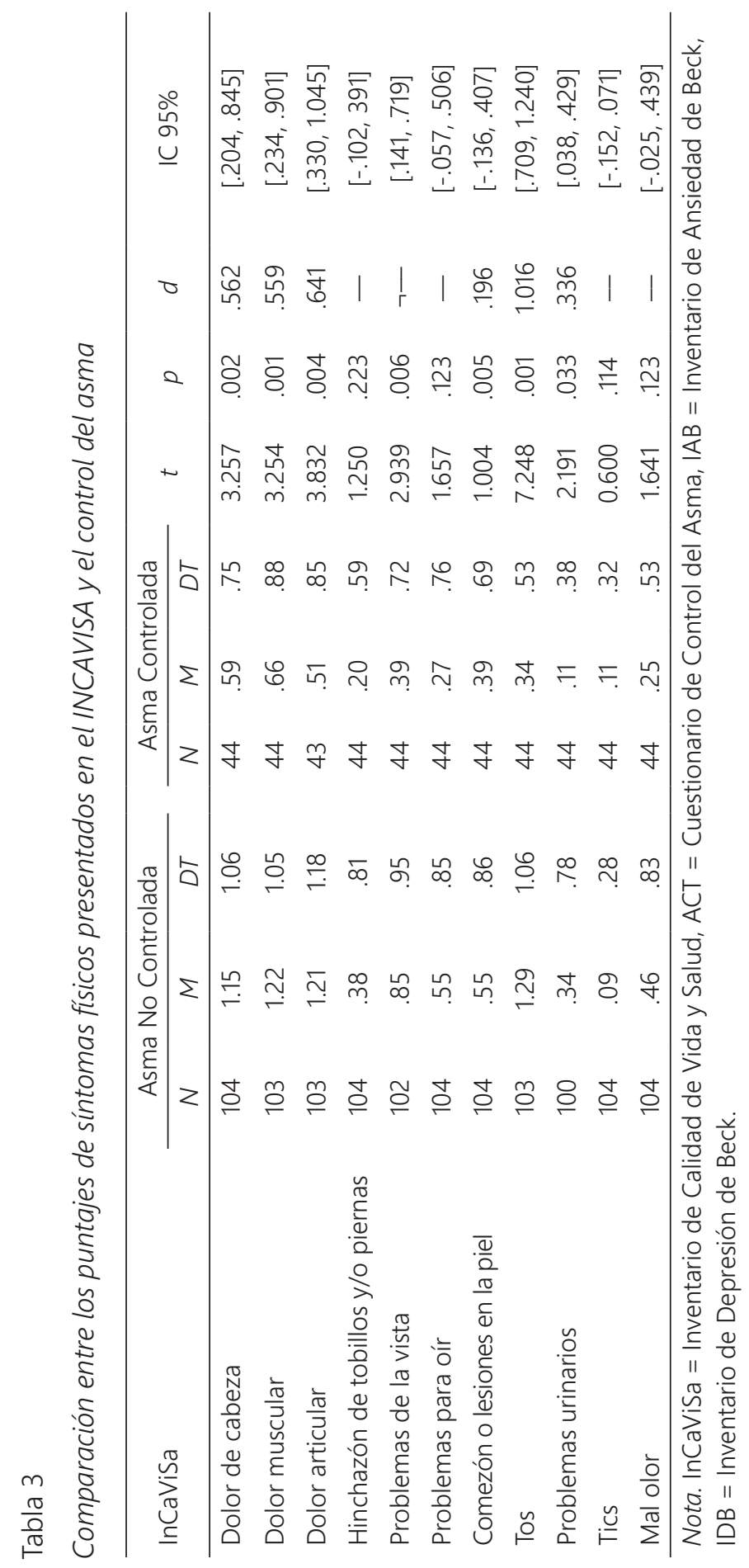

Actualidades en Psicología, 35(131), 2021, 35-51 
Tabla 4

Correlaciones entre sub-escalas del InCaViSa

\begin{tabular}{|c|c|c|c|c|c|c|c|c|c|c|c|}
\hline & 1 & 2 & 3 & 4 & 5 & 6 & 7 & 8 & 9 & 10 & 11 \\
\hline 1. $P$ & 1 & & & & & & & & & & \\
\hline 2. DF & $.21^{\star \star}$ & 1 & & & & & & & & & \\
\hline 3. A & $.50^{\star \star}$ & $.29^{\star *}$ & 1 & & & & & & & & \\
\hline 4. $P C$ & $43^{\star *}$ & $.35^{\star *}$ & $.69^{* \star}$ & 1 & & & & & & & \\
\hline 5. FC & $.42^{* *}$ & .29 & .40 & $.42^{\star *}$ & 1 & & & & & & \\
\hline 6. AT & $.53^{\star \star}$ & .20 & $.54^{\star \star}$ & $.47^{\star \star}$ & $.43^{* *}$ & 1 & & & & & \\
\hline 7. F & $.13^{\star *}$ & $.26^{\star *}$ & $.25^{\star \star}$ & $.30^{\star \star}$ & $.18^{*}$ & $.19^{\star}$ & 1 & & & & \\
\hline 8. TL & $.50^{\star \star}$ & $.43^{\star \star}$ & .59 ** & $.57^{* \star}$ & $.37 * \star$ & $.61^{\star \star}$ & $.20^{\star}$ & 1 & & & \\
\hline 9. VC & $.52^{\star *}$ & $.45^{\star \star}$ & $.78^{\star \star}$ & $.64^{\star \star}$ & $.43^{* *}$ & $.60^{\star *}$ & .34 & $.77^{\star \star}$ & 1 & & \\
\hline 10. DM & $.27^{\star \star}$ & .60 & $.31^{\star \star}$ & $.24^{\star *}$ & .10 & .11 & .07 & $.29^{\star *}$ & $.27^{\star \star}$ & 1 & \\
\hline 11. RM & $.12^{*}$ & .04 & .14 & .08 & .10 & .01 & .12 & $.18^{*}$ & $.21^{\star}$ & $.52^{\star \star}$ & 1 \\
\hline 12. RS & .28 & $.40^{\star *}$ & $.43^{\star \star}$ & $.34^{\star *}$ & $.24^{\star *}$ & $.24^{\star \star}$ & $.43^{* *}$ & $.37^{\star \star}$ & $.43^{* \star}$ & $.27^{\star \star}$ & .03 \\
\hline
\end{tabular}

Nota. 1. $P=$ Preocupaciones, $2 . D F=$ Desempeño físico, 3. $A=$ Aislamiento, $4 . P C=$ Percepción corporal, 5. $F C=$ Funciones cognitivas, $6 . A T=$ Actitud ante el tratamiento, 7. $F=$ Familia, 8. $T L$ = Tiempo libre, 9. $V C=$ Vida cotidiana, 10. $D M=$ Dependencia médica, 11. $R M=$ Relación con el médico, 12. $R S=$ Redes sociales.

${ }^{*} p<.05,{ }^{* *} p<.01,{ }^{* *} p<.001$ (dos colas)

Tabla 5

Correlaciones con las sub-escalas de aislamiento, percepción corporal, vida cotidiana, total del InCaViSa, IAB, IDB y ATC

\begin{tabular}{|c|c|c|c|c|c|c|c|}
\hline & 1 & 2 & 3 & 4 & 5 & 6 & 7 \\
\hline 1. Aislamiento & 1 & & & & & & \\
\hline 2. Percepción Corporal & $.69^{* \star}$ & 1 & & & & & \\
\hline 3. Vida Cotidiana & $.78^{\star \star}$ & $.64^{\star *}$ & 1 & & & & \\
\hline 4. InCaViSa & $.78^{\star \star}$ & $.57^{\star \star}$ & $.62^{\star \star}$ & 1 & & & \\
\hline 5. ACT & $.23 * *$ & $.24^{\star \star}$ & $.39 * *$ & $.29 * *$ & 1 & & \\
\hline 6. IAB & $-.45^{\star \star}$ & $-.38^{\star *}$ & $.51^{\star \star}$ & $-.40^{* \star}$ & $-.44^{\star \star}$ & 1 & \\
\hline 7. IDB & $-.64^{\star \star}$ & $-.54^{\star \star}$ & $-.65^{\star \star}$ & $-.39 * *$ & $-.30^{\star \star}$ & $.51^{\star \star}$ & 1 \\
\hline
\end{tabular}

Nota. 4. InCaViSa = Puntaje total del Inventario de Calidad de Vida y Salud, 5. ACT = Cuestionario de Control del Asma, 6. IAB = Inventario de Ansiedad de Beck, 7. IDB= Inventario de Depresión de Beck. Las áreas del InCaViSa restantes presentaron correlaciones de nulas a bajas, por lo que no se representan en la tabla.

${ }^{*} p<.05,{ }^{* *} p<.01,{ }^{* * *} p<.001$ (dos colas) 


\section{Discusión}

La aportación principal del presente trabajo radicó en comparar las áreas que evalúa el InCaViSa, así como depresión y ansiedad entre pacientes con asma controlada y no controlada. Además, el objetivo particular era conocer las correlaciones entre dichas variables.

Un primer punto para explorar en los resultados es que en gran proporción (70\%) los participantes reportaron un asma no controlada (por medio del auto-reporte). Esto es similar con lo encontrado por IImarinen et al. (2019) quienes reportan en su investigación un 66\% de pacientes con asma no controlada por medio del ACT y cercano a los porcentajes que presenta la GINA (2021) que expone que más del 50\% de los pacientes no están controlados.

En lo referente a la evaluación del apego al tratamiento, un dato a resaltar es que en la autoevaluación de apego la calificación promedio fue de 7.8 (de 10 puntos a obtener). No se reportaron diferencias entre los niveles de control. Entre las probables explicaciones a este fenómeno se propone explorar la deseabilidad social, o incluso, explorar si en el caso de esta muestra en particular hay una mala autopercepción del apego al tratamiento, las creencias personales, y creencias culturales, entre otras (Shahin et al., 2019).

Sería conveniente estudiar si entre la población mexicana con asma, existen discrepancias entre la función pulmonar y la percepción de apego al tratamiento, ya que en investigaciones se ha asentado que los pacientes con asma suelen hacer una evaluación errónea de su apego a las recomendaciones del tratamiento y de su función pulmonar que incluye evaluar previamente si reconocen las medidas de control para su tratamiento y cómo llevarlas a cabo (Steele et al., 2012). Si el paciente puede evaluar de forma objetiva su apego, serviría para auxiliarlo en la toma de decisiones en cuanto a su tratamiento médico y control de la enfermedad, lo que podría repercutir en su calidad de vida.

En referencia a las áreas contenidas en el InCaViSa, se encontró que las áreas en las que se reportaron diferencias significativas con un tamaño del efecto moderado fueron desempeño físico, aislamiento, tiempo libre y vida cotidiana. El grupo de usuarios con asma no controlada resultó con menores puntuaciones en cada una de dichas áreas. Estas áreas tienen en común la ejecución de actividades físicas o sociales, ya sea que requieren esfuerzo o demandan energía; por lo que se sugiere que la ejecución de actividades instrumentales es el parámetro más afectado en la calidad de vida de los pacientes con asma no controlada.

Las áreas señaladas son similares a las encontradas por Ilmarinen et al. (2019), quienes reportaron que el asma no controlada se asoció con una reducción en las áreas de CdV general como, actividades usuales o diarias, depresión, distrés, vitalidad y actividad sexual; y estas áreas también se relacionan con la instrumentalización de actividades físicas.

Los hallazgos de esta investigación contrastan con los encontrados con pacientes con hipertensión arterial, quienes reportan mayores daños en las áreas de redes sociales y funciones cognitivas, siendo estas áreas las más afectadas debido a los efectos secundarios de los medicamentos empleados para el tratamiento de la hipertensión (Riveros, Ceballos et al., 2005). En el caso de pacientes con asma, los resultados proponen que el pobre control de la enfermedad aqueja significativamente la CdV y esta alteración se traduce a áreas que incluyen actividades diarias y ocio. Esto es similar a lo mencionado por Stucky et al. (2015) que exponen que la habilidad de participar en roles y actividades sociales son de los factores que tienen más peso para los individuos para el control de la enfermedad asmática. 
Otro aspecto relevante es que los pacientes con asma no controlada presentaron mayor intensidad de molestias por la tos, el cual es un síntoma característico del asma. Una posible explicación a los demás síntomas (dolor de cabeza, dolor muscular) podrían ser consecuencia del mal control de la enfermedad.

En cuanto a las variables psicológicas evaluadas se encontraron diferencias entre los niveles de ansiedad y depresión, medidos a través de los inventarios de Beck, en el que las puntuaciones desfavorecieron al grupo de pacientes con asma no controlada. En este sentido, se sostiene la premisa que han aportado la gran mayoría de investigaciones al tema y esta es que el estado del control del asma se relaciona con en el estado anímico del paciente (González-Freire et al., 2019).

El área de aislamiento y actividades en la vida cotidiana correlacionaron con el IDB, estas áreas tienen componentes que son comportamientos característicos de la depresión (como son el retraimiento en relaciones sociales, anhedonia, percepción corporal inadecuada, entre otras). En las recomendaciones de aplicación e interpretación del InCaViSa se hace hincapié en evaluar en depresión a aquellos pacientes que obtienen puntuaciones bajas en el área de aislamiento (Riveros et al., 2009), para así poder dirigir la atención psicológica a resolver los síntomas depresivos.

La relación entre el estado de ánimo depresivo y el mal control asmático puede ser explicado desde la perspectiva conductual, ya que se asume que el estado de ánimo genera que el paciente adquiera o no, conductas de autocuidado, o a través de los efectos que estos estados de ánimo producen sobre el sistema nervioso e inmune (Lavoie et al., 2006). Otra variable para considerar es que el aislamiento y estado de ánimo pueden ser resultado de la sobreprotección dentro del entorno familiar, esta consiste en preocupación excesiva que limita al paciente a hacer actividades por sí solos (Caso, 2006).

En el caso de la ansiedad, no se obtuvo una asociación con ninguna de las áreas evaluadas, sin embargo, un punto importante es que el grupo de pacientes con asma no controlada obtuvo una media cercana al punto de corte para considerar ansiedad clínicamente importante. Este hallazgo debería tomarse con precaución ya que un factor a tener en cuenta es que en ocasiones los síntomas fisiológicos de ansiedad medidos por el BAI y los síntomas característicos del asma son confundidos por los pacientes, lo que puede llevar a que se atribuya ansiedad cuando no la hay (Guillén \& González-Celis, 2019).

Una de las principales justificaciones para el desarrollo del presente trabajo fue el poder generar propuestas de intervención derivadas de la información obtenida, por lo tanto, en adelante se desarrollan sugerencias al respecto. Como primer punto, aunque no formó parte del objetivo central en el estudio, es relevante destacar que la percepción del apego al tratamiento no difiere dependiendo el nivel del control del asma y los usuarios suelen puntuarse casi con calificaciones perfectas. En este sentido se podrían en agregar medidas de auto-reporte, complementarias al inventario ACT, como el Asthma Control Questionnaire (Juniper et al., 1999); otra opción de medidas subjetivas pueden ser los registros o carnés de adherencia (Caballero-Suárez et al., 2009) y de principalmente, valerse de medidas objetivas de función pulmonar por medio de técnicas como la flujometría y espirometría.

Referente a las sugerencias para mejorar la $\mathrm{CdV}$, las intervenciones psicológicas deben ir encaminadas a la promoción de bienestar en las áreas que se reportaron afectación. Primordialmente, se sugiere atender las áreas de desempeño físico y tiempo libre, que fueron las más disminuidas para los usuarios con asma no controlada. Para todas las áreas, Riveros et al. (2009) proporcionan una serie de recomendaciones con pasos y 
técnicas a seguir para mejorar el bienestar del sujeto. En el caso del desempeño físico, los autores del InCaViSa infieren que, al tener puntuaciones bajas, la capacidad para realizar esfuerzos físicos se encuentra deteriorada y también, podrían estar refiriendo poca tolerancia al dolor, reactividad cardiovascular y respiración deficiente. También, algunos autores refieren desconocimiento de la enfermedad (Eisenberg et al., 2020) y por tanto del efecto benéfico que puede tener el ejercicio con prescripción en estos pacientes (Hernández, 2020; Hansen et al., 2020; Lucas et al., 2018). Por lo tanto, las intervenciones psicológicas pudieran ser dirigidas a favorecer el conocimiento y beneficio de la activación conductual de estos pacientes, acorde a su prescripción individualizada.

Para el área de tiempo libre, se debe atender a la percepción del paciente sobre su enfermedad en el desempeño y disfrute de sus actividades de ocio. Las intervenciones psicológicas podrán dirigirse a evaluar la afectación de las actividades del paciente a partir del diagnóstico de enfermedad, cuáles fueron y promover la búsqueda de actividades que pueda llevar acabo el paciente (Riveros et al., 2009), se puede hacer uso de las técnicas previamente señaladas, como psicoeducación, solución de problemas, reestructuración cognitiva (Guillén et al., 2020). Además, es conveniente agregar técnicas como desarrollo de habilidades sociales de comunicación, asertividad, negociación, entre otras (García \& Palacios, 2006).

Por último, se hace énfasis en el área de aislamiento, ya que esta área se correlacionó fuertemente con depresión, por lo que las intervenciones deberán promover el reforzamiento y creación de redes de apoyo, junto con el desarrollo de las habilidades sociales. Estas mismas técnicas podrían reducir la sintomatología depresiva de forma indirecta. No se debe olvidar la sugerencia de evaluar una probable sobreprotección por parte de la familia que esté contribuyendo al aislamiento.Relacionado con lo anterior, una gran área de oportunidad es precisamente el componente de la familia, que fue el área en la que ambos grupos (pacientes controlados y no controlados) puntuaron más alto. Esto parece proponer que el paciente percibe un apoyo emocional adecuado por parte de los miembros de su familia. Es aceptable generar talleres que refuercen el apoyo emocional brindado por la red de apoyo primaria, pero también se promueva el apoyo instrumental/conductual. Se debe contar con talleres dirigidos a familiares que promuevan psicoeducación del asma, el tratamiento médico, qué hacer en caso de crisis asmática y comportamientos de la familia para beneficiar el control del asma y autonomía del paciente.

Dentro de las limitaciones del estudio se señalan que la selección de la muestra es no probabilística por conveniencia, lo que conlleva a impedir la generalización de los datos. Debe recordarse que eran pacientes que recurrían a una clínica especializada en el control del asma, lo que puede jugar un papel importante, ya que son pacientes que se encuentran con especialistas y tratamientos más dirigidos a su tipo de enfermedad, además, pueden percibir que sus respuestas influirán en su atención médica.

Esta investigación se vale del auto reporte, lo cual conlleva problemas propios de este tipo de evaluación como la deseabilidad social, fallas en la memoria del paciente y errores escalares. Otra de las limitaciones de este estudio es que no se realizó una evaluación de la adherencia al tratamiento con una herramienta específica y estandarizada y de forma longitudinal. Situación que se puede subsanar en futuras investigaciones.

Finalmente, se considera dirigir las intervenciones para fomentar comportamientos que busquen beneficiar la calidad de vida y salud emocional de los pacientes con asma. 
Por ejemplo, acompañar al paciente en la búsqueda de actividades que le produzcan placer, auxiliar en actividades que requieran algún esfuerzo físico importante y promocionar ideas racionales que apoyen el apego al tratamiento.

\section{Referencias}

Caballero-Suárez, N., Fernández-Vega, M., Salas-Hernández, J., Chapela-Mendoza, R., \& Sánchez-Sosa, J. (2009). Efectos de una intervención psicológica cognitivoconductual sobre la adhesión terapéutica y la ansiedad en pacientes asmáticos. Revista Española de Asma, 1(4), 6-15.

Caso, M. A. (2006). La sobreprotección parental como factor asociado en el asma bronquial en niños. Psicología y Salud, 16(1), 33-39. https://www.redalyc.org/pdf/291/29116104.pdf

Coban, H., \& Ediger, D. (2018). Control of asthma, quality of life, anxiety, and depression symptoms among Turkish patients with asthma. Electronic Journal of General Medicine, 15(5), 71-101. https://doi.org/10.29333/ejgm/91403

Coban, H., \& Aydemir, Y. (2014). The relationship between allergy and asthma control, quality of life, and emotional status in patients with asthma: a cross-sectional study. Allergy, Asthma \& Clinical Immunology, 10(67), 78-90. https://doi. org/10.1186/s13223-014-0067-4

Ekici, A., Ekici, M., Kara, T., Keles, H., \& Kocyigit, P. (2006). Negative mood and quality of life in patients with asthma. Quality of Life Research, 15(1), 49-56. https://doi. org/10.1007/s11136-005-8869-y

Eisenberg, S. R., Jelalian, E., Farrow, M., Kopel, S. J., Vehse, N., Mitchell, P., Dunsiger, S., \& Koinis-Mitchell, D. (2020). Perceptions of Asthma and Exercise, and Associations with Weight Status and Asthma Morbidity in Urban Children. Academic pediatrics, 20(1), 55-62. https://doi.org/10.1016/j.acap.2019.07.001

Fernández-Ballesteros, R. (1997). Quality of life: Concept and assessment. In J. G. Adair, D. Belanger., \& K. L. Dion (Eds.), Advances in Psychological Science. Social, Personal and Cultural Aspects (pp. 387-406). Psychological Press.

García, I. G., \& Palacios, L. (2006). La educación en paciente con asma y EPOC. En J. G. Soto (Ed), Manual de diagnóstico y terapéutica en Neumología (pp. 339-348). Ergon. https://www.neumosur.net/files/EB04-33\%20educacion.pdf

González-Barcala, F. J., de la Fuente, R., Tafalla, M., Nuevo, J., \& Camaño-Isorna, F. (2012). Factors associated with health-related quality of life in adults with asthma. A cross-sectional study. Multidisciplinary Respiratory Medicine, 7(2), 2-10. https:// doi.org/10.1186/2049-6958-7-32

González-Freire, B., Vázquez, I., \& Pértega-Díaz, S. (2019). The relationship of psychological factors and asthma control to health-related quality of life. Journal of Allergy and Clinical Immunology: In Practice. 8(1), 197-207 https://doi.org/10.1016/j. jaip.2019.07.009

Guillén, D. B. C., \& González-Celis, R. A. L. (2019). Propiedades psicométricas del Inventario de Ansiedad de Beck en adultos asmáticos mexicanos. Psicología y Salud, 29(1), 5-16. https://psicologiaysalud.uv.mx/index.php/psicysalud/ article/view/2563/4471

Actualidades en Psicología, 35(131), 2021, 35-51 
Guillén, D. B. C., González-Celis Rangel, A. L., Cruz-Peralta, M., Mendoza-Madrigal, V. A., Reyes-Jarquín, K., \& Bautista-Díaz, M. L. (2020). Efectos de una intervención cognitivo-conductual para mujeres con síntomas de depresión y asma no controlada. Revista Latinoamericana de Medicina Conductual, 10(2). http:// revistas.unam.mx/index.php/rlmc/article/view/79730

Iniciativa Global del Asma. (2021). Global Strategy for Asthma Management and Prevention. https:// ginasthma.org/wp-content/uploads/2021/05/GINA-Main-Report-2021-V2-WMS.pdf

Hansen, E., Pitzner-Fabricius, A., Toennesen, L. L., Rasmusen, H. K., Hostrup, M., Hellsten, Y., Backer, V., \& Henriksen, M. (2020). Effect of aerobic exercise training on asthma in adults: a systematic review and meta-analysis. The European Respiratory Journal, 56(1). https://doi.org/10.1183/13993003.00146-2020

Hernández, J. Q. (2020). Ejercicio físico como posible tratamiento de apoyo no farmacológico para pacientes con asma grave no alérgica: Artículo de reflexión. Movimiento Científico, 14(1), 27-32. https://revmovimientocientifico.ibero.edu.co/article/view/1798

Hossny, E., Caraballo, L., Casale, T., El-Gamal, Y., \& Rosenwasser, L. (2017). Severe asthma and quality of life. World Allergy Organization Journal, 10(1), 28-32. https://doi. org/10.1186/s40413-017-0159-y

Ilmarinen, P., Juboori, H., Tuomisto, L., Niemela, O., Sintonen, H., \& Kankaanranta, H. (2019). Effect of asthma control on general health-related quality of life in patients diagnosed with adult-onset asthma. Scientific Reports, 9(1), 1-11. https:// doi.org/10.1038/s41598-019-52361-9

Jansson, S.-A., Axelsson, M., Hedman, L., Leander, M., Stridsman, C., \& Rönmark, E. (2016). Subjects with well-controlled asthma have similar health-related quality of life as subjects without asthma. Respiratory Medicine, 120, 64-69. https://doi. org/10.1016/j.rmed.2016.09.019

Juárez, L. A. (2010). Evaluación de una intervención psicológica para mejorar la adherencia terapéutica sobre la calidad de vida de pacientes con asma [Tesis de maestría, Universidad Nacional Autónoma de México]. TESISUNAM. http://132.248.9.195/ ptd2014/anteriores/0713547/Index.html

Jurado, S., Villegas, M., Méndez, L., Rodríguez, F., Loperena, V., \& Varela, R. (1998). La estandarización del Inventario de Depresión de Beck para los residentes de la Ciudad de México. Salud Mental, 21(3), 26-31. http://www.revistasaludmental.mx/ index.php/salud_mental/article/view/706

Juniper, E. F., O'Byrne, P. M., Guyatt, G. H., Ferrie, P. J., \& King, D. R. (1999). Development and validation of a questionnaire to measure asthma control. The European Respiratory Journal, 14(4), 902-907. https://doi.org/10.1034/j.1399-3003.1999.14d29.x

Larenas-Linnemann, D., Salas-Hernández, J., Vázquez-García, J. C., Ortiz-Aldana, I., Fernández-Vega, M., del Río-Navarro, B. E., Cano-Salas, M., Luna-Pech, J., Ortega-Martell, J., Romero-Lombard, J., López-Estrada, E., Villaverde-Rojas, J., \& Mayorga-Butrón, J. (2017). Guía Mexicana del Asma. Revista Alergia México, 64(1), s11-s128. https://www.medigraphic.com/pdfs/neumo/nt-2017/nts171a.pdf 
Lavoie, K., Bacon, S., Barone, S., Cartier, A., Ditto, B., \& Labreque, M. (2006). What is worse for asthma control and quality of life: Depressive disorders, anxiety disorders or both? Chest, 130, 1039-1047. https://doi.org/10.1378/chest.130.4.1039

Lucas, J. A., Moonie, S., Hogan, M. B., \& Evans, W. N. (2018). Efficacy of an exercise intervention among children with comorbid asthma and obesity. Public Health, 159, 123-128. https://doi.org/10.1016/j.puhe.2018.02.014

Lomper, K., Chudiak, A., Uchmanowicz, I., Rosinczuk, J., \& Jankowska-Polanska, B. (2016). Effects of depression and anxiety on asthma-related quality of life. Pneumonologia i Alergologia Polska, 84(4), 212-221. https://doi.org/10.5603/PiAP.2016.0026

Méndez-Guerra, G., Salas-Hernández, J., Vargas, H., Pérez-Chavira, R., León-Munguía, L., Franco-Martínez, S., \& Chapela-Mendoza, R. (2003). Calidad de vida en pacientes asmáticos mexicanos. Revista del Instituto Nacional de Enfermedades Respiratorias, 16(4), 234-242. https://www.medigraphic.com/pdfs/iner/in-2003/in034f.pdf

Nathan, R. A., Sorkness, C. A., Kosinski, M. Schatz, M., Li, T., Marcus, P., Murray, J., \& Pendergraft, T. (2004). Development of the asthma control test: A survey for assessing asthma control. Journal of Allergy and Clinical Immunology, 113, 59-65. https://doi.org/10.1016/j.jaci.2003.09.008

Riveros, A., Ceballos, G., Laguna, R., \& Sánchez-Sosa, J. J. (2005). El manejo psicológico de la hipertensión esencial: efectos de una intervención cognitivo-conductual. Revista Latinoamericana de Psicología, 37(3), 493-507. http://pepsic.bvsalud.org/

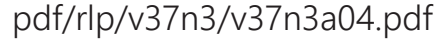

Riveros, A., Cortazar-Palapa, J., Alcazar, L. F., \& Sánchez-Sosa, J. J. (2005). Efectos de una intervención cognitivo-conductual en la calidad de vida, ansiedad, depresión y condición médica de pacientes diabéticos e hipertensos esenciales. International Journal of Clinical and Health Psychology, 5(3), 445-462. https://www.redalyc.org/ articulo.oa?id=33705302

Riveros, A., Sánchez-Sosa, J. J., \& del Águila, M. (2009). Inventario de Calidad de Vida y Salud (InCaViSa). Manual Moderno.

Robles, R., Varela, R., Jurado, S., \& Páez, F. (2001). Versión mexicana del Inventario de Ansiedad de Beck: propiedades psicométricas. Revista Mexicana de Psicología, 18(2), 211-218

Sánchez-Sosa, J. J., \& González-Celis, A. L. (2006). Evaluación de la calidad de vida desde la perspectiva psicológica. En V. E. Caballo (Ed.), Manual Para la Evaluación Clínica de los Trastornos Psicológicos (pp. 473-491). Ediciones Pirámide.

Shahin, W., Kennedy, G. A., \& Stupans, I. (2019). The impact of personal and cultural beliefs on medication adherence of patients with chronic illnesses: a systematic review. Patient Preference and Adherence, 13, 1019-1035. https://doi.org/10.2147/ppa.s212046

Steele, A. M., Meurel, A. E., Millard, M. W., \& Ritz, T. (2012). Discrepancies between lung function and asthma control: asthma perception and association with demographics and anxiety. Allergy \& Asthma Proceedings, 33(6), 500-5017. https://doi.org/10.2500/aap.2012.33.3611

Stucky, B.D., Sherbourne, C. D., Edelen, M. O., \& Eberhart, N. K. (2015). Understanding asthmaspecific quality of life: moving beyond asthma symptoms and severity. European Respiratory Journal, 46(3), 680-687. https://doi.org/10.1183/09031936.00225014 
Uchmanowicz, I., Panaszek, B., Uchmanowicz, B., \& Rosińczuk, J. (2016). Sociodemographic factors affecting the quality of life of patients with asthma. Patient Preference and Adherence, 10, 345. https://doi.org/10.2147/ppa.s101898

Urzúa, M. A., \& Caqueo-Urízar, A. (2012). Calidad de vida: Una revisión teórica del concepto. Terapia Psicológica, 30(1), 61-71. https://scielo.conicyt.cl/pdf/terpsicol/v30n1/art06.pdf 